\title{
A Multi-Band, High Bandwidth, Short Duration Linear Chirp Generation IC for Phased-Array Applications
}

\author{
J. Velner*, E.A.M. Klumperink*, B. Nauta*, F.E. van Vliet*† \\ *University of Twente, 7500 AE, Enschede, The Netherlands ${ }^{\dagger}$ TNO, P.O. Box 96864, 2509 JG, The Hague, The Netherlands
}

\begin{abstract}
This work presents a fully integrated multi-band frequency synthesizer that is capable of creating wide-band linear frequency sweeps of very short duration. The main aim is to create a flexible waveform generation system, requiring distribution of a low frequency reference rather than RF signals. By using a phase-locked loop, frequency multiplication (rather than shifting) occurs. This allows for a narrow band and low frequency reference signal, allowing digital reference generation and thus providing a large degree of flexibility. Chirps with a bandwidth of $1 \mathrm{GHz}$ (in X-band) and duration as short as $10 \mu \mathrm{s}$ can be created. The chip can be operated in either pulsed or CW mode. The small external size of the package, which measures $5 \times 5 \mathrm{~mm}^{2}$, means that local $\mathrm{RF}$ generation is possible even in $\mathrm{X}$-band arrays with small antenna element pitch.

Index Terms-Phase locked loops, Phased arrays, Ultra wideband radar, Signal generators, BiCMOS integrated circuits,
\end{abstract}

\section{INTRODUCTION}

Pulse-Doppler phased-array radar systems generally use a centralized signal generator that produces a pulsed waveform with very high spectral purity. This signal is up-converted and distributed (or vice versa) over the active antenna aperture. For future phased-arrays with a high level of reconfiguration or with multiple waveforms that are simultaneously transmitted, such as MIMO systems, this approach poses strong constraints [1]. In view of these systems, sources that can be integrated at individual antenna element level are required, locked only to a central frequency or timing reference for coherency reasons.

In this work, a system is presented which can serve as a fully integrated RF source for a single array element. Where frequency up-conversion is often achieved by means of mixers, we propose to use a phase-locked loop instead. This has the advantage of multiplying, rather than shifting, the input (reference) frequency. This means that the reference signal can be narrow band, as well as relatively low frequency. This means that these signals can be created digitally with high flexibility. In the most flexible case each chip would have its own DSP, allowing distribution of a single, constant frequency, digital clock while providing a fully configurable array. Another advantage of this approach is that it is no longer required to generate and distribute a large number of RF signals.

The IC can generate signals in X-band, covering 7.2 to 12.2 GHz. In this band it can create chirps with durations down to $10 \mu \mathrm{s}$ and bandwidths of up to $1 \mathrm{GHz}$ with an RMS frequency error of $3.088 \mathrm{MHz}$. By means of frequency division (naturally occurring in the PLL's divider) signals in C, S- and L-band can be created too, with proportionally lower

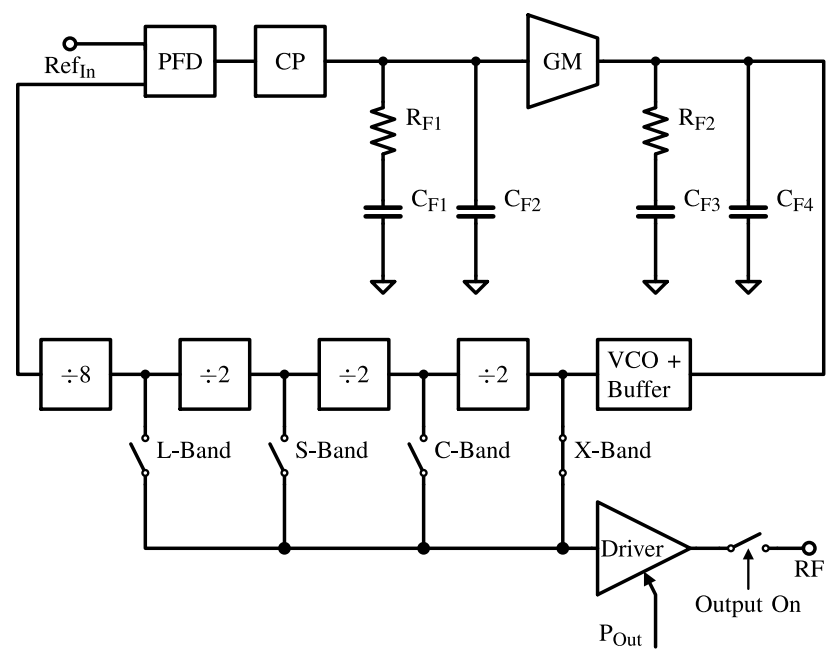

Fig. 1. System Architecture

bandwidth and frequency error. It features an output driver which can deliver a differential signal with up to $4.9 \mathrm{dBm}$ into a $100 \Omega$ load (dropping to $-0.7 \mathrm{dBm}$ at the highest output frequency). This driver can also fully disable the output, allowing for the generation of pulsed signals.

This paper is divided into five sections. Section II describes the design on a system-level. Measurement results are given in section III. This section is divided into some general results, a subsection treating chirp linearity and a subsection giving FMCW and range-Doppler results. A comparison with other work is made in section IV. The final conclusions are treated in section $\mathrm{V}$.

\section{System OVERVIEW}

A block level overview of the system can be found in figure 1. This figure depicts a phase-locked loop, which is a feedback system which operates on phase. The reader will recognize a PLL with one extra filter stage. This means one extra integrator in the loop filter compared to most PLLs. The system depicted here therefore of type III, with three integrators in the loop (two in the filter and one in the conversion of control voltage to output frequency, which is the time-integral of phase, by the VCO). In terms of dynamic behavior, a type II system can track a signal with first order behavior with no static error and a type III system, can do the same for a second order signal. This means that, ideally, this 
PLL can track a signal with second order phase versus time characteristics without static errors (perfect linear frequency ramp).

The system can create frequency chirps in several frequency bands. It has two VCOs, which are designed to cover the Xband with an overlap of over $1 \mathrm{GHz}$ to allow wideband chirps without switching from one VCO to the other. As can be seen in figure 1, the output can alternatively be connected to one of three divider outputs, dividing the aforementioned frequency range by two, four or eight. This allows operation in C-, S- or L-band. The total division factor of the divider is 64 , thus a reference signal between $125 \mathrm{MHz}$ and $187.5 \mathrm{MHz}$ is required to cover the full X-band.

The signal is then fed to a differential output driver which can be set to any one of 16 possible output power levels (on a linear voltage scale for ease of implementation) and which provides $50 \Omega$ on both outputs (100 $\Omega$ differentially). This termination is resistive in order to provide large bandwidth. The driver can also be switched off completely in order to create a pulsed output, functionality which is depicted as a switch in the output in figure 1 . The output driver was designed to deliver $-18 \mathrm{dBm}$ to $6 \mathrm{dBm}$ differentially.

Readers who are interested in some of the circuit aspects of this work are referred to [2], which describes the actual implementation.

\section{iII. Measurement Results}

Measurements have been performed in order to verify the functionality of the IC. The ultimate frequency range over which the VCOs operate is $7.2 \mathrm{GHz}$ to $10.1 \mathrm{GHz}$ for the first VCO and $8.8 \mathrm{GHz}$ to $12.2 \mathrm{GHz}$ for the second, an overlap of $1.3 \mathrm{GHz}$. The output measured output power was $-17.9 \mathrm{dBm}$ to $4.9 \mathrm{dBm}$ in the lower frequency bands. This value drops to $-22.6 \mathrm{dBm}$ to $-0.7 \mathrm{dBm}$ for frequencies high in the $\mathrm{X}$ band. Since the IC is in a sealed package, the output cannot be measured directly at the bondpads. However the simulated bandwidth of the PA is over $16 \mathrm{GHz}$, thus it is expected that this drop is mainly caused by the bondwires and the first section of the transmission lines on the test PCB, which were shaped to fit to the IC package.

\section{A. Chirp Linearity}

The ability of the IC to generate fast frequency ramps has been verified. An example of this for a static signal and two chirps with a pulse length of $10 \mu$ s is depicted in figure 2 . These measurements were made by sampling the L-band output of the IC with a $40 \mathrm{GSa} / \mathrm{s}$ oscilloscope and post processing in Matlab to extract the frequency. A bandpass filter with cut-off frequencies at $1 \mathrm{GHz}$ and $2 \mathrm{GHz}$ and $60 \mathrm{~dB}$ out of band suppression was used to suppress any out of band spurs which give rise to frequency errors. The RMS frequency error for both chirps was found to be $386 \mathrm{kHz}$ and that of the constant frequency was $391 \mathrm{kHz}$, confirming the independence of frequency error of chirp rate. That been said, there are still some spurs left inside the frequency band of the chirp, which are believed to be the main cause of the remaining

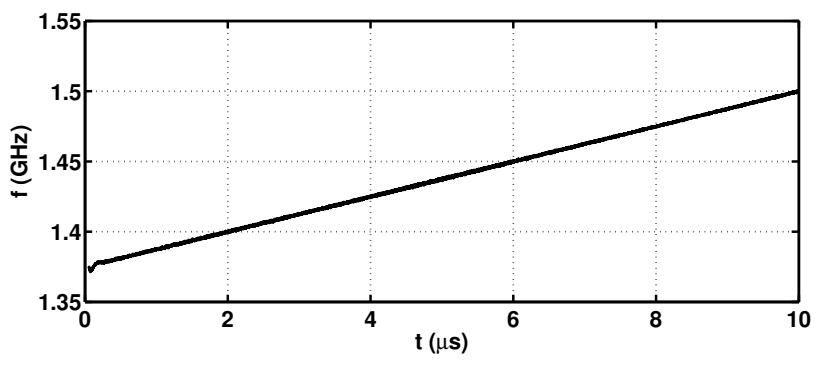

(a) $125 \mathrm{MHz}$ in $10 \mu \mathrm{s}$

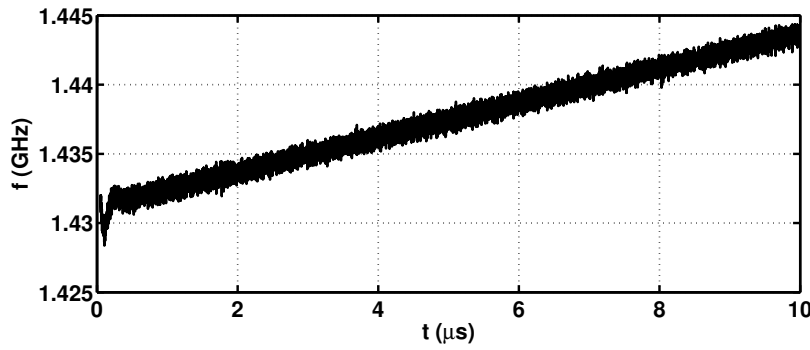

(b) $12.5 \mathrm{MHz}$ in $10 \mu \mathrm{s}$

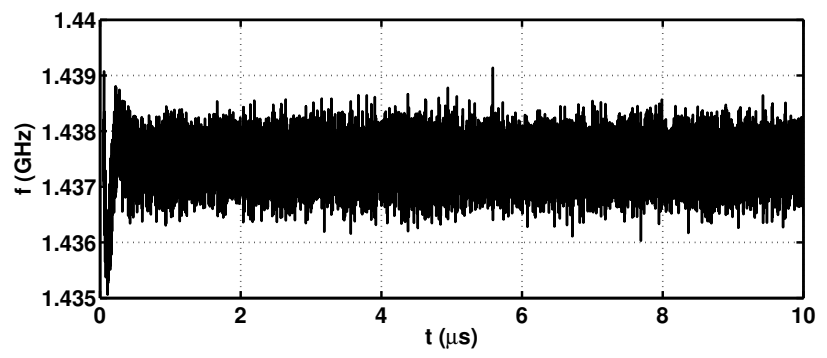

(c) Constant Frequency

Fig. 2. Frequency vs. Time

frequency errors. The higher frequency bands could not be measured directly with available equipment, but the errors can be inferred from the errors at L-band by simple multiplication (inverse of the frequency divider operation). This gives an RMS frequency error of $3.088 \mathrm{MHz}$ for a bandwidth of $1 \mathrm{GHz}$ in X-band.

\section{B. FMCW and Range-Doppler Measurements}

FMCW measurements have been performed by connecting the output of the chip to a programmable delay line and mixing the output of this line with the non-delayed output of the chip. Figure 3 shows the results of some of these measurements. Figure 3a shows the beat frequency spectrum of a $375 \mu$ s, $300 \mathrm{MHz}$ bandwidth sweep in L-band applied to a delay line that simulates a target at $28 \mathrm{~km}$. Figure $3 \mathrm{~b}$ shows the result for the same chirp in S-band with a target at $8 \mathrm{~km}$ and figure $3 \mathrm{c}$ shows the result of a $375 \mu$ s chirp over $900 \mathrm{MHz}$ in X-band, also with a target at $8 \mathrm{~km}$. Note that in order to create a bandwidth of $300 \mathrm{MHz}$ in L-band, a bandwidth of $2.4 \mathrm{GHz}$ is required in X-band. This is only possible using the high 


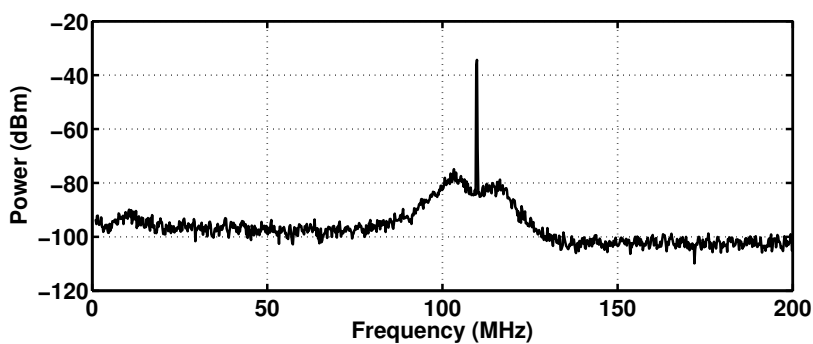

(a) L-band Measurement, Target at $28 \mathrm{~km}$

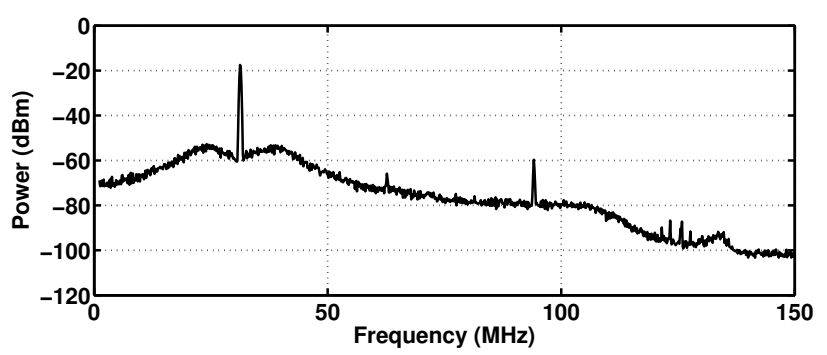

(b) S-band Measurement, Target at $8 \mathrm{~km}$

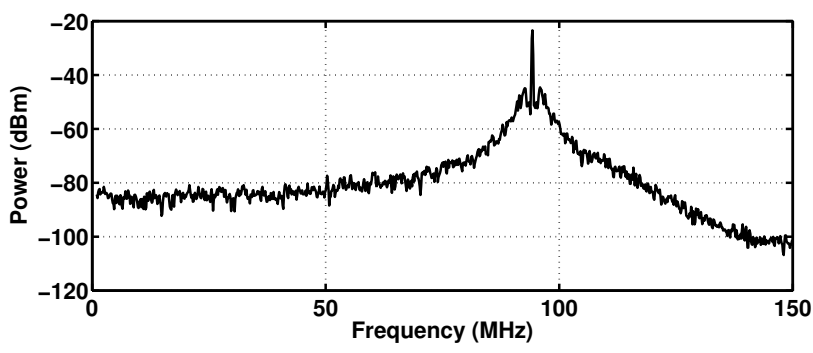

(c) X-band Measurement, Target at $8 \mathrm{~km}$

Fig. 3. FMCW Measurements

frequency $\mathrm{VCO}$ and therefore only in the $8.8 \mathrm{GHz}$ to $12.2 \mathrm{GHz}$ range. It will also give rise to more spurs in the band of the chirp. This is no problem for FMCW range measurements, as the spurs will always be at $\mathrm{f}_{\text {Ref }}$ distance from the beat note, but it will be detrimental for pulsed signals where the bandwidth of the filter will now include several spurs.

The phase noise of the PLL can be clearly seen in these figures, with skirts around the peak that indicate the bandwidth of the loop at that particular frequency. The additional peaks at $60 \mathrm{MHz}$ and $90 \mathrm{MHz}$ in figure $3 \mathrm{~b}$ are caused by the second and third order harmonics. These give rise to (small) signals that sweep at two and three times the sweep rate of the fundamental, and thus produce two and three times the frequency difference after a fixed delay. The third order harmonic is larger than the second order harmonic because the output of the chip approaches a square wave in the lower frequency bands.

Several X-and L-band FMCW measurements were logged using a sampling oscilloscope. These data were used to create range-Doppler representations of the measurements. The first step in this process is to take an FFT over the data in each sweep, giving frequency (and thus range) information. This

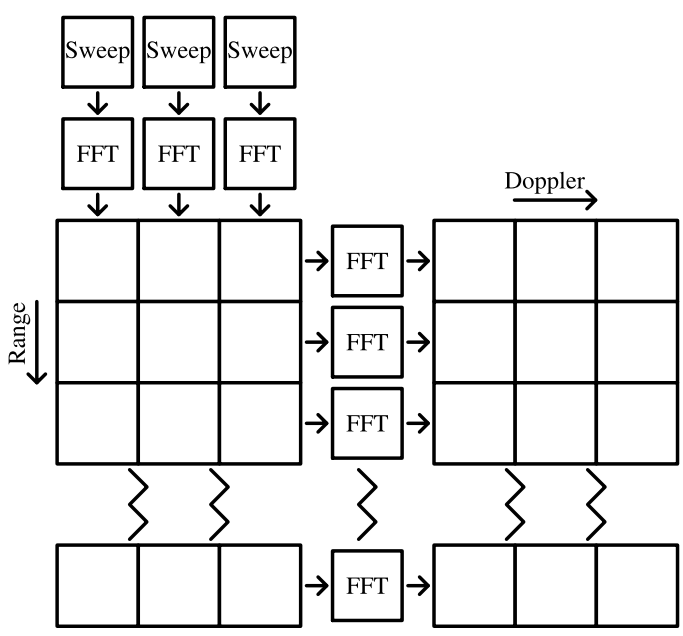

Fig. 4. Graphical Representation of Range-Doppler Processing

result is stored in a 2-D matrix where each column contains the range data for one sweep. Subsequently FFTs over the rows of this matrix are taken, creating a new matrix with range information along its columns and Doppler information along its rows. A graphical representation of this procedure is displayed in figure 4 .

A slow moving target will give rise to a negligible Doppler shift and thus small range error during one sweep. Its changing distance to the radar will however give rise to small phase shifts over a longer period of time. Such phase shifts become evident after range-Doppler processing, producing a peak in a non-zero Doppler bin. This also means that a range-Doppler plot displays the phase stability over several frequency ramps when a static target is emulated, such as was done here. A linear phase drift will cause the signal to be in a non-zero Doppler bin, and a random phase drift will cause it to be smeared out over several Doppler bins.

Figure 5 shows the results of range-Doppler processing over 32 sweeps in both $\mathrm{S}$ - and $\mathrm{X}$-band (targets again at $8 \mathrm{~km}$ ). For both cases the peak is narrow, containing significant power in three range and three Doppler bins after which it falls off rapidly. The fact that the response is not smeared out along the Doppler axis indicate good phase correspondence between subsequent sweeps. Note that the range is less than $8 \mathrm{~km}$. This is mainly due to extra delays in the reference path of the setup that were not calibrated out, as these measurements were performed to characterize the IC and not to build an actual radar. The plot for the X-band configuration shows more noise than the S-band setup. This is due to the extra frequency (and hence phase noise) division that occurs when lower bands are output. It also has a spur like component along the range axis (which is hidden behind the main lobe in figure $5 \mathrm{~b}$, the origin of which is as yet unknown.

\section{COMPARISON TO OTHER WORK}

As the authors are not aware of previous publications of pulsed systems with this level of integration on silicon, a 
TABLE I

COMPARISON TABLE

\begin{tabular}{|c|c|c|c|c|c|c|c|}
\hline Type & $\begin{array}{c}{[3]} \\
\text { FMCW }\end{array}$ & $\begin{array}{c}{[4]} \\
\text { FMCW }\end{array}$ & $\begin{array}{c}{[5]} \\
\text { FMCW }\end{array}$ & $\begin{array}{c}{[6]} \\
\text { FMCW }\end{array}$ & $\begin{array}{c}{[7]} \\
\text { FMCW }\end{array}$ & $\begin{array}{l}\text { This Work } \\
\text { Pulsed }\end{array}$ & \\
\hline Technology & $65 \mathrm{~nm}$ CMOS & $90 \mathrm{~nm}$ CMOS & $65 \mathrm{~nm} \mathrm{CMOS}$ & SiGe Bipolar ${ }^{a}$ & Board & $250 \mathrm{~nm}$ BiCMOS & \\
\hline Active Area & 1.05 & 6.8 & 1.7 & $3^{a}$ & $?$ & 3 & $\mathrm{~mm}^{2}$ \\
\hline Supply Voltage & 1.2 & 1.2 & 1.2 & $5^{a}$ & ? & 2.5 & $\mathrm{~V}$ \\
\hline Power Consumption & $188^{b}$ & $406^{b}$ & 152 & $488^{a}$ & $?$ & $380^{c}$ & $\mathrm{~mW}$ \\
\hline Output Frequency & 75.6 to 76.3 & 78.1 to 78.8 & 82.1 to 83.8 & 67.2 to 92.8 & 76 to 78 & 7.2 to $12.2^{d}$ & $\mathrm{GHz}$ \\
\hline Chirp BW & 0.7 & 0.7 & 1.5 & 25.6 & 2 & $1^{d}$ & $\mathrm{GHz}$ \\
\hline Fractional Bandwidth & 1.3 & 1.3 & 2.3 & 32 & 2.6 & 10 & $\%$ \\
\hline RMS Frequency Error & $<300^{e}$ & $1050^{e}$ & 180 & ? & $?$ & $3088^{d}$ & $\mathrm{kHz}$ \\
\hline Chirp Time & $0.5^{f, g}$ & $0.25^{f, g}$ & 0.5 to $5^{f}$ & $4^{f}$ & $1^{f, g}$ & $\geq 0.01$ & $\mathrm{~ms}$ \\
\hline Output Power & 5.1 & -2.8 & $?$ & -3 to -1 & 10 & $-1 \overline{7} .9$ to 4.9 & $\mathrm{dBm}$ \\
\hline Phase Noise@1 MHz & -85.3 & -85 & -84 & -90 & $?$ & -86.0 to $-81.4^{d}$ & $\mathrm{dBc} / \mathrm{Hz}$ \\
\hline
\end{tabular}

${ }^{a}$ Core IC; requires external ICs ${ }^{b}$ Synthesizer $+\mathrm{TX}{ }^{c}$ Max power, CW Mode ${ }^{d}$ In X-band, divide by 2, 4 or 8 for lower bands

${ }^{e}$ Including turn-around points ${ }^{f}$ Only increasing freq. part ${ }^{g}$ Lowest value reported

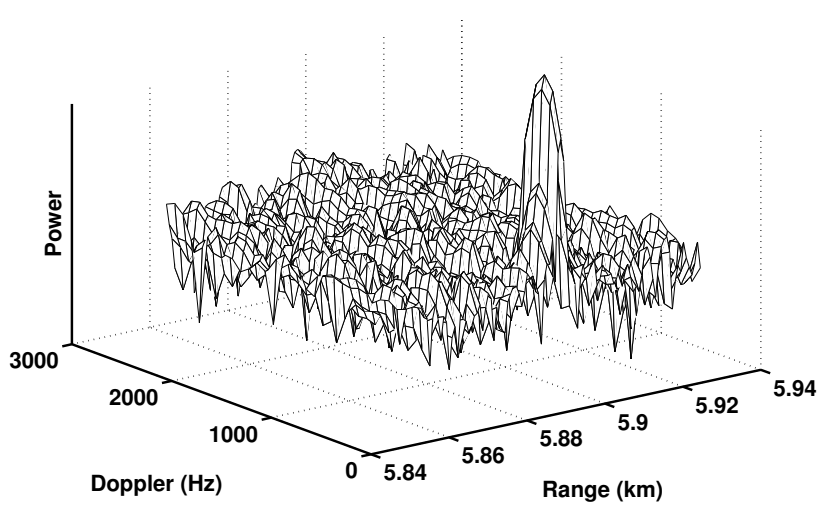

(a) S-band Measurement, Target at $8 \mathrm{~km}$

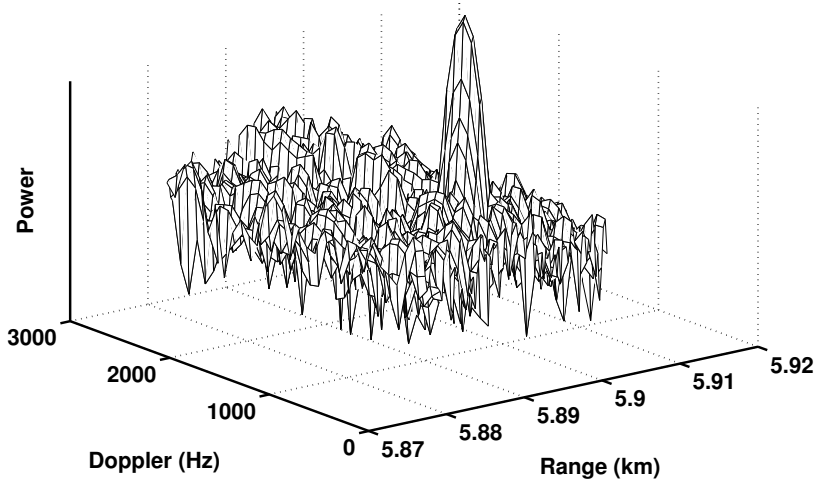

(b) X-band Measurement, Target at $8 \mathrm{~km}$

Fig. 5. FMCW Range-Doppler Processing

comparison is made with several FMCW systems. The real benefits, however, are present for fast-chirping pulse-Doppler systems. This comparison can be found in table I. As can be seen, the frequency error can be improved upon, but the chirp rate is by far the highest reported. Fractional bandwidth is surpassed only by [6], which has a far lower level of integration than this work.

\section{Conclusions}

The feasibility of a single chip solution for the creation of very fast and wide-band linear frequency chirps is proven in this work. A $5 \times 5 \mathrm{~mm}^{2}$ device was created that can generate $1 \mathrm{GHz}$ chirps in X-band with durations as low as $10 \mu$ s from a relatively low frequency, easily distributed, reference signal. On-chip frequency division allows for chirp generation in C-, $\mathrm{S}$ - and L-band as well. An output driver that provides output power control, $\mathrm{CW}$ and pulsed signal generation and wideband matching is included on the IC. A step towards future phasedarrays with a high level of reconfiguration, or with multiple waveforms that are simultaneously transmitted, has been made.

\section{ACKNOWLEDGEMENTS}

The authors would like to thank NXP Semiconductors for silicon donation. Further thanks are extended to Maurice van Wanum and Lex de Boer at TNO for their insights concerning the design and layout of the circuit. Finally the authors would like to thank Henk de Vries, Gerard Wienk, Mark Oude Alink, Bram Verhoef and Erik Olieman for invaluable assistance during the functional testing and characterization phases of the circuit.

\section{REFERENCES}

[1] J. Li, P. Stoica, MIMO Radar Signal Processing, Wiley, 2008.

[2] J. Velner, et al., "Multi-Band Linear Chirp Generation Based on a Type-III PLL," MTT Digest, 2013.

[3] Y. Li, et al., "A Fully Integrated 77GHz FMCW Radar System in $65 \mathrm{~nm}$ CMOS," ISSCC Dig. Tech. Papers, pp. 216-217, February 2010.

[4] T. Mitomo, et al., "A $77 \mathrm{GHz} 90 \mathrm{~nm}$ CMOS Transceiver for FMCW Radar Applications," IEEE J. Solid-State Circuits, vol. 45, no. 4, pp. 928-937, April 2010.

[5] H. Sakurai, et al., "A 1.5GHz-Modulation-Range 10ms-ModulationPeriod $180 \mathrm{kHz}$ RMS-Frequency-Error 26MHz-Reference Mixed-Mode FMCW Synthesizer for mm-Wave Radar Application," ISSCC Dig. Tech. Papers, pp. 292-294, February 2011.

[6] N. Pohl, et al., "An Ultra-Wideband $80 \mathrm{GHz}$ FMCW Radar System Using a SiGe Bipolar Transceiver Chip Stabilized by a Fractional-N PLL Synthesizer," IEEE Trans. Microw. Theory Tech., vol. 60, no. 3, pp. 757765, March 2012.

[7] C. Pfeffer, et al., "An IQ Modulator Based Heterodyne 77-GHz FMCW Colocated MIMO Radar System," MTT Digest, pp. 1-3, June 2012. 